Pacific Journal of Mathematics

A COMPARISON OF THE RELATIVE UNIFORM TOPOLOGY
AND THE NORM TOPOLOGY IN A NORMED RIESZ SPACE 


\title{
A COMPARISON OF THE RELATIVE UNIFORM TOPOLOGY AND THE NORM TOPOLOGY IN A NORMED RIESZ SPACE
}

\author{
L. C. MOORE, JR.
}

It is well-known that if a normed Riesz space $(L, \rho)$ is $\rho$-complete, i.e., a Banach lattice, then the relative uniform topology and the norm topology are the same. Under weaker conditions the two topologies show some measure of agreement. In particular if $L$ has (i) the local $\sigma$-property (a property weaker than both the $\sigma$-property and local completeness) and (ii) the property that for every ideal $A$ the norm closure of $A$ equals the set of limit points of relatively uniformly convergent sequences of elements of $A$, then every sequence $u_{n} \downarrow 0$ with $\rho\left(u_{n}\right) \rightarrow 0$ is a relatively uniformly convergent sequence. (This generalizes a theorem of Luxemburg and Zaanen.) However conditions (i) and (ii) are not sufficient to imply that the relative uniform topology and the norm topology agree on order intervals. Examples are given illustrating increasing degrees of agreement of the two topologies.

Let $L$ be a Riesz space, $\left\{x_{n}: n \in N\right\}$ be a sequence in $L, x \in L$, and $v \in L^{+}$, then $\left\{x_{n}\right\}$ converges $v$-uniformly to $x$, written $x_{n} \rightarrow x$ $\left(v\right.$-unif.), if there is a sequence $\left\{\alpha_{n}\right)$ of real numbers such that $\alpha_{n} \downarrow 0$ and $\left|x_{n}-x\right| \leqq \alpha_{n} v$ for each $n$ in $N$. A sequence $\left\{x_{n}\right\}$ of elements of $L$ is said to converge relatively uniformly to $x \in L$, written $x_{n} \rightarrow x$ (r.u.), if $x_{n} \rightarrow x$ ( $v$-unif.) for some $v$ in $L^{+}$. If $S$ is a subset of $L$, define $S^{\prime r u}$ to be the set of $x$ in $L$ such that there exists a sequence $\left\{x_{n}\right\} \subseteq S$ with $x_{n} \rightarrow x(r . u$.). A subset $S$ of $L$ is said to be relatively uniformly closed if $S=S^{\prime r u}$, and the relatively uniformly closed sets are exactly the closed sets for a topology, the relative uniform topology $\tau_{r u}$, on $L$. For an arbitrary subset $S$ of $L$ the set $\bar{S}^{\text {rru }}$ is called the relative uniform closure of $S$ and is denoted by $\bar{S}^{r u}$.

The relative uniform topology and its relation to the order structure on $L$ have been investigated in some detail. (See [2].) We note here that although $S \subseteq S^{\prime r u} \subseteq \bar{S}^{r u}$ for every set $S$ in $L$, it is not necessarily true that $S^{\prime r u}=\bar{S}^{r u}$. Recall that $L$ is said to have the $\sigma$-property if every sequence of elements of $L$ is contained in a principal ideal, i.e., given any sequence $\left\{u_{n}\right\}$ in $L^{+}$, there is a sequence $\left\{\lambda_{n}\right\}$ of positive numbers and an element $u \in L^{+}$such that $u_{n} \leqq \lambda_{n} u$ for each $n \in N$. In particular if $L$ has a strong unit, it has the $\sigma$-property. 
The following is a theorem of T. Chow [2]:

Theorem 1. Let $L$ be an Archimedean Riesz space. Then the following conditions are equivalent:

(i) $L$ has the o-property.

(ii) $S^{\prime r u}=\bar{S}^{r u}$ for every subset $S$ of $L$.

(iii) $S^{\prime r u}=\bar{S}^{r u}$ for every convex subset of $L$.

Now let $\rho$ be a Riesz norm on $L$. It is well-known that if $(L, \rho)$ is a Banach lattice then the norm topology $\tau_{\rho}$ and the relative uniform topology coincide. Since $\tau_{r u}$ is determined intrinsically by the order structure of $L$, this shows that the norm topology is intrinsic for a Banach lattice and that, in this case, $L$ has the $\sigma$-property. Even if $L$ is not $\rho$-complete, it is of interest to determine the relation between the norm topology and the relative uniform topology. Luxemburg and Zaanen presented a result of this type at the 1973 Oberwolfach Conference on Riesz Spaces. Their theorem is:

THEOREM 2. If $L$ has the $\sigma$-property and $A^{\prime r u}=\bar{A}^{\rho}$ for each ideal $A$ in $L$, then $u_{n} \downarrow_{\rho} 0$ implies $u_{n} \rightarrow 0$ (r.u.).

Here $u_{n} \downarrow_{\rho} 0$ means $u_{n} \downarrow 0$ (in order) and $\rho\left(u_{n}\right) \rightarrow 0$. The main element in the proof is due to Luxemburg and appears as Lemma 1 in the present paper. In Theorem 2 the assumption that $L$ has the $\sigma$-property guarantees a certain "richness" of elements in $L$ (in lieu of $L$ being norm complete). As we shall see the assumption that $L$ has the $\sigma$-property may be replaced by the assumption that $L$ is locally complete (i.e., every order interval is $\rho$-complete). Indeed we need assume only that $(L, \rho)$ has the local $\sigma$-property, a property defined below which is weaker than both the $\sigma$-property and local completeness (Theorems 4 and 5).

Definition 1. A normed Riesz space $(L, \rho)$ is said to satisfy the local $\sigma$-property if for every $u \in L^{+}$, for every $0 \leqq v_{k} \uparrow$ in $L$ and for every $\varepsilon>0$, there exist $w \in L$ and real numbers $\beta_{k}>0$ for $k=1,2, \cdots$ such that $0 \leqq w \leqq u, \rho(u-w)<\varepsilon$ and if $0 \leqq z \leqq w$ and $z \leqq \alpha v_{k}$ for some $k \in N$ then $z \leqq \alpha \beta_{k} u$.

Roughly $(L, \rho)$ has the local $\sigma$-property if locally (within order intervals) $L$ almost has the $\sigma$-property.

We include here for reference the following definition from [1]:

Definition 2. Let $(L, \rho)$ be a normed Riesz space and let $\hat{L}$ be 
the $\rho$-completion of $L$. An element $u \in \hat{L}^{+}$is called a lower element if there is a sequence $\left\{u_{n}\right\} \subseteq L^{+}$such that $u_{n} \downarrow_{\rho} u$, and $u$ is called an upper element if there is a sequence $\left\{u_{n}\right\} \subseteq L^{+}$such that $u_{n} \uparrow_{\rho} u$.

The author wishes to express his appreciation to W. A. J. Luxemburg for many helpful conversations about the results and examples in this paper.

Main results. We describe the main results here and give the proofs and examples in the next section. Consider the following conditions on a normed Riesz space $(L, \rho)$ :

(1) $\bar{A}^{\rho}=\bar{A}^{r u}$ for every ideal $A$ in $L$.

(2) $\bar{A}^{\rho}=A^{\prime r u}$ for every ideal $A$ in $L$.

(3) $u_{n} \downarrow_{\rho} 0$ implies $u_{n} \rightarrow 0$ (r.u.).

(4) $\bar{S}^{\rho}=S^{\prime r u}$ for every solid set $S$ in $L$.

(5) $\tau_{r u}=\tau_{\rho}$ on order intervals.

(6) $\tau_{r u}=\tau_{\rho}$ on principal ideals.

(7) $\tau_{r u}=\tau_{\rho}$ on principal bands.

(8) $\tau_{r u}=\tau_{\rho}$ on $L$.

(9) $L$ is $\rho$-complete.

THEOREM 3. Using the notation above we have:

(i ) $(9) \Rightarrow(8) \Rightarrow(7) \Rightarrow(6) \Rightarrow(5) \Leftrightarrow(4) \Rightarrow(3) \Rightarrow(2) \Rightarrow(1)$.

(ii) Except for $(4) \Rightarrow(5)$, none of the reverse implications hold.

THEOREM 4. If $(L, \rho)$ has the local $\sigma$-property, then $(2) \Rightarrow(3)$, i.e., if $(L, \rho)$ has the property that $\bar{A}^{\rho}=A^{\prime r u}$ for all ideals $A$ in $L$, then $u_{n} \downarrow_{\rho} 0$ implies $u_{n} \rightarrow 0$ (r.u.).

THEOREM 5. If $L$ has the $\sigma$-property or if in the $\rho$-completion of $L$ every lower element is an upper element (in particular if $(L, \rho)$ is locally complete) then $(L, \rho)$ has the local $\sigma$-property.

\section{Proofs and examples.}

Proof of Theorem 3. The only implications which are not trivial are $(4) \Leftrightarrow(5)$.

$((5) \Rightarrow(4))$. Assume $(L, \rho)$ satisfies (5), i.e., $\tau_{\rho}=\tau_{r u}$ on order intervals. We have to show that $\bar{S}^{\rho}=S^{\prime r u}$ for every solid set $S$ in $L$. Let $S$ be a solid set and let $x \in \bar{S}^{\rho}$. Then we have a sequence $\left\{x_{n}\right\}$ in $S$ such that $x_{n} \rightarrow x(\rho)$. Since $x_{n}^{+} \rightarrow x^{+}(\rho)$ and $x_{n}^{-} \rightarrow x^{-}(\rho)$ and since $\left|\inf \left(x_{n}^{+}, x^{+}\right)-\inf \left(x_{n}^{-}, x^{-}\right)\right| \leqq\left|x_{n}\right|$ and $\left|\inf \left(x_{n}^{+}, x^{+}\right)-\inf \left(x_{n}^{-}, x^{-}\right)\right| \leqq$ $|x|$, we may assume $\left|x_{n}\right| \leqq|x|$. Then by assumption there is a subsequence of $\left\{x_{n}\right\}$ which converges relatively uniformly to $x$. Thus 
$x \in S^{\prime r u}$ and $S^{\prime r u} \supseteqq \bar{S}^{\rho}$. Since the reverse inclusion holds in any normed Riesz space, we are done.

$((4) \Rightarrow(5))$. Assume $(L, \rho)$ satisfies $(4)$, i.e., $\bar{S}^{\rho} \equiv S^{\prime r u}$ for every solid set $S$ in $L$. In order to show that $\tau_{\rho}=\tau_{r u}$ on order intervals, it is sufficient to show that if $w \in L^{+}$, if $\left\{u_{n}\right\}$ is a sequence in $[0, w]$, and if $u_{n} \rightarrow u(\rho)$, then there is a subsequence $\left\{u_{n_{k}}\right\}$ such that $u_{n_{k}} \rightarrow u(r . u$.$) .$

Suppose there is a subsequence $\left\{u_{n_{k}}\right\}$ such that $u_{n_{k}} \leqq u$ for all $k$. In this case we may assume $u_{n_{k}} \neq u$ for all $k$. Let $S$ be the solid set generated by $\left\{u_{n_{k}}: k \in N\right\}$. Since $u \in \bar{S}^{\rho}$ there exists by assumption a sequence $\left\{v_{j}\right\}$ in $S$ so that $v_{j} \rightarrow u(r . u$.$) . Pick k_{j}$ for each $j \in N$ so that $0 \leqq v_{j} \leqq u_{n_{k_{j}}}$. Since $u_{n_{k}} \neq u$ for any $k$, we may assume that the sequence of integers $\left\{n_{k_{j}}\right\}$ is strictly increasing. Then we have that $0 \leqq u-u_{n_{k_{j}}} \leqq u-v_{j}$ for all $j$, so $u_{n_{k_{j}}} \rightarrow u(r . u$.). Similarly if there is a subsequence $\left\{u_{n_{k}}\right\}$ such that $u_{n_{k}} \geqq u$ for all $k$, then arguing as above (with $w-u$ replacing $u$ ) we again conclude that $\left\{u_{n}\right\}$ has a subsequence converging relatively uniformly to $u$. Now we may assume $u_{n} \not \leq u$ and $u \not \equiv u_{n}$ for all $n$. Again let $S$ be the solid set generated by $\left\{u_{n}: n \in N\right\}$. Again $u \in \bar{S}^{\rho}$ so there exists a sequence $\left\{v_{j}\right\}$ in $S$ so that $v_{j} \rightarrow u(r . u$.$) . If for some n$ and infinitely many $j$ we have $v_{j} \leqq u_{n}$ then $u_{n} \geqq u$ which is a contradiction. Thus we may assume that we have a strictly increasing sequence $\left\{n_{j}\right\}$ of integers so that $v_{j} \leqq u_{n_{j}}$ for each $j$. Now repeating the argument with the sequence $\left\{w-u_{n_{j}}\right\}$, we find that we may assume that for each $j \in N$ there exists $z_{j}$ such that $0 \leqq z_{j} \leqq w-u_{n_{j}}$ and $z_{j} \rightarrow w-u$ $(r . u$.$) . Then w-z_{j} \geqq u_{n_{j}}$ for all $j$ and $w-z_{j} \rightarrow u(r . u$.$) . Thus$

$$
\begin{aligned}
\left|u-u_{n_{j}}\right| & =\left(u-u_{n_{j}}\right)^{+}+\left(u-u_{n_{j}}\right)^{-} \\
& \leqq\left(u-v_{j}\right)^{+}+\left(u-\left(w-z_{j}\right)\right)^{-} \\
& \leqq\left|u-v_{j}\right|+\left|u-\left(w-z_{j}\right)\right|
\end{aligned}
$$

and so $u_{n_{j}} \rightarrow u(r . u$.$) .$

Part (ii) of Theorem 3 follows from Examples 1-7 below.

EXAMPLE 1. $\quad((1) \nsim(2))$. In this example we have $\bar{A}^{\rho}=\bar{A}^{r u}$ for every ideal $A$ of $L$ but we do not have $\bar{A}^{\rho}=A^{\prime r u}$ for every ideal $A$ of $L$.

In the Riesz space of all sequences let $K$ be the ideal generated by the sequences $z^{k}$ where $z^{k}(j)=j^{k}$ for each $k=1,2, \cdots$. Let $K_{n}=K$ for $n=1,2, \cdots$ and let $L$ be the ideal in $\prod_{n \in N} K_{n}$ consisting of those $x$ such that there exist $m$ and $r$ in $N$ and $\lambda \in R$ (all depending on $x$ ) such that if $n>m$ then $\left|x_{n}(j)\right| \leqq \lambda n^{r}$ for all $j \in N$. Note that if $x \in L$ then for $n$ sufficiently large $x_{n}(\cdot) \in \ell_{\infty}$. We define a norm $\rho$ on $L$ by defining 


$$
\rho_{n}(x)=\sum_{j=1}^{\infty} \frac{\left|x_{n}(j)\right|}{j^{j}} \text { for } x \in L \text { and } n \in N .
$$

For each $x \in L$ there exist $m$ and $r \in N$ and $\lambda \in R$ such that if $n>m$ we have

$$
\rho_{n}(x) \leqq \lambda n^{r} \sum_{j=1}^{\infty} \frac{1}{j^{j}}
$$

Thus we may define $\rho$ by

$$
\rho(x)=\sum_{n=1}^{\infty} \frac{\rho_{n}(x)}{n^{n}} .
$$

(i) We show next that every relatively uniformly closed ideal is a band and hence norm closed. This implies (1) since the relative uniform closure of an ideal is again an ideal. (Note that this shows that the particular choice of Riesz norm $\rho$ is unimportant.)

Let $A$ be a relatively uniformly closed ideal in $L$. Let $M=$ $\left\{(n, j): x_{n}(j) \neq 0\right.$ for some $\left.x \in A\right\}$. We assert that $A$ consists exactly of those $x \in L$ such that $x_{n}(j)=0$ if $(n, j) \notin M$. It then follows that $A$ is a band. So assume $x \in L$ such that $x_{n}(j)=0$ if $(n, j) \notin M$. For each $m \in N$ define $x^{(m)}$ by

$$
x_{n}^{(m)}(j)=\left\{\begin{array}{ll}
x_{n}(j) & \text { if } n \leqq m \\
0 & \text { if } n>m
\end{array} .\right.
$$

For each $m$ and $p$ in $N$ define $x^{(m, p)}$ by

$$
x_{n}^{(m, p)}(j)= \begin{cases}x_{n}(j) & \text { if } n \leqq m \text { and } j \leqq p \\ 0 & \text { otherwise }\end{cases}
$$

Since for each $m$ and $p$ in $N$ the set $\left\{(n, j): x_{n}^{(m, p)}(j) \neq 0\right\}$ is finite, it follows that $x^{(m, p)} \in A$. We assert that for each $m$ we have that $x^{(m, p)} \stackrel{p}{\rightarrow} x^{(m)}(r . u$.$) and that x^{(m)} \rightarrow x(r . u$.$) . Thus x \in A$ since $A$ is relatively uniformly closed.

Now if $m \in N$ we have

$$
x_{n}^{(m)}(j)-x_{n}^{(m, p)}(j)= \begin{cases}0 & \text { if } j \leqq p \text { or } n>m \\ x_{n}(j) & \text { otherwise }\end{cases}
$$

There exist constants $\beta_{n} \in R$ and $k_{n} \in N$ for $n \leqq m$ such that $\left|x_{n}(j)\right| \leqq \beta_{n} j^{k_{n}}$ all $j \in N$. Define $v \in L$ by

$$
v_{n}(j)= \begin{cases}j^{k_{n}+1} & \text { for } n \leqq m \\ 0 & \text { for } n>m .\end{cases}
$$

Since $x_{n}^{(m)}(j)-x_{n}^{(m, p)}(j)=0$ for $j \leqq p$ we have $\left|x_{n}^{(m)}(j)-x_{n}^{(m, p)}(j)\right| \leqq$ 
$\left(\beta_{n} / p\right) j^{k_{n}+1}$ for all $j$ and $p$. Hence $\left|x^{(m)}-x^{(m, p)}\right| \leqq(\beta / p) \quad v$ where $\beta=\max \left(\beta_{1}, \beta_{2}, \cdots, \beta_{m}\right)$. Thus $x^{(m, p)} \rightarrow x^{(m)}(v$-unif. $)$ and $x^{(m)} \in A$ for all $m$.

Finally pick $m_{0}$ and $r \in N$ and $\lambda \in R^{+}$such that $\left|x_{n}(j)\right| \leqq \lambda n^{r}$ for $n \geqq m_{0}$. Then $x^{(m)} \rightarrow x$ ( $w$-unif.) where $w \in L$ is defined by $w_{n}(j)=n^{r+1}$ for all $j$.

(ii) To complete the example we exhibit an ideal $I$ such that $I^{r u} \neq \bar{I}^{r u}$. Let $I$ be the ideal of all $x \in L$ such that for some $m$ and $p$ (depending on $x$ ) $x_{n}(j)=0$ if $n>m$ or $j>p$. By the argument above we see that $\bar{I}^{r u}=L$. Now if $u \in I^{\prime r u}$ then for $n$ sufficiently large, $u_{n}($.$) is the uniform limit of sequences with finite support, i.e.,$ for $n$ sufficiently large $\lim _{j} u_{n}(j)=0$. Thus the element $e$ defined by $e_{n}(j)=1$ for all $n$ and $j$ is in $\bar{I}^{r u}=L$ but is not in $I^{r u}$.

EXAMPLE 2. $((2) \nRightarrow(3))$. In this example we have $\bar{A}^{\rho}=A^{\prime r u}$ for all ideals $A$ but there exists a sequence $u_{n} \downarrow_{\rho} 0$ which does not converge relatively uniformly.

Pick $g \in L_{1}([0,1])$ such that $g$ is continuous on $(0,1], g(x)>0$ for all $x \in(0,1]$, and $\lim _{x \rightarrow 0^{+}} f(x)=\infty$. Let $L$ be the Riesz subspace of $L_{1}([0,1])$ consisting of all $f$ such that for some $\delta(f) \in R^{+}$and $\gamma(f) \in R$ we have $f(x)=\gamma(f) g(x)$ for all $0 \leqq x<\delta(f)$. Define a Riesz norm $\rho$ on $L$ by setting

$$
\rho(f)=\|f\|_{1}+|\gamma(f)| \text {. }
$$

(i) We show that if $A$ is an ideal in $L$ then $A^{r u}=\bar{A}^{\rho}$. Suppose $0 \leqq h \in \bar{A}^{\rho}$. We may assume there exists a sequence $\left\{f_{n}\right\} \cong A^{+}$such that $f_{n} \uparrow h$ and $\rho\left(h-f_{n}\right) \rightarrow 0$. If $\gamma(h)=0$, then there exists $\delta=\delta(h)>0$ such that $h(x)=0$ for $x \in[0, \delta)$. Then since $L_{1}([\delta, 1])$ is norm complete, there exists $w \in L_{1}([0,1])$ such that $w(x)=0$ for $x \in[0, \delta)$ and $f_{n} \rightarrow h$ (w-unif.).

Assume $\gamma(h)>0$. Then there exists $m \in N$ such that $\gamma\left(f_{m}\right)>0$. For some $\delta>0$

$$
h(x)=\frac{\gamma(h)}{\gamma\left(f_{m}\right)} f_{m}(x) \text { for } x \in[0, \delta] .
$$

Now we can replace $h$ by $h-\inf \left(h,\left(\gamma(h) / \gamma\left(f_{m}\right)\right) f_{m}\right)$ and argue as above.

(ii) Next we exhibit a sequence $\left\{f_{n}\right\}$ in $L$ such that $f_{n} \downarrow_{\rho} 0$ but $f_{n} \nrightarrow 0(r . u$.$) . Let f_{0}=g$. Define $f_{n}$ by induction so that

(a) $f_{n}$ is continuous on $(0,1]$,

(b) $0 \leqq f_{n} \leqq f_{n-1}$,

(c) $f_{n}(1 / k)=1 / k f_{0}(1 / k) k=1,2, \cdots, n$,

(d) $f_{n}(x)=1 /(n+1) g(x), x \in(0,1 /(n+1)]$,

(e) $\left\|f_{n}\right\|_{1} \leqq 1 /(n+1)\|g\|_{1}$. 
Then $\rho\left(f_{n}\right) \leqq 1 /(n+1)+1 /(n+1)$ for all $n$, hence $f_{n} \downarrow_{\rho} 0$.

Now if $h \in L^{+}$and $\gamma(h)>0$ there exists $\delta>0$ such that for all $x \in(0, \delta]$ we have $h(x)=\gamma(h) g(x)$. If $k \in N$ is sufficiently large that $1 / k<\delta$, then $k \gamma(h) f_{n}(1 / k)=h(1 / k)$ for all $n \geqq k$. Thus $f_{n} \nrightarrow 0$ (h-unif.) and it follows that $f_{n} \not 0$ (r.u.).

(iii) We show that $L$ does not have the local $\sigma$-property. This follows immediately from Theorem 4. However we give here a direct argument. Let $u=g$ and define functions $v_{k}$ in $L$ for $k=1,2 \ldots$ so that

(a) $v_{k}$ is continuous on $[0,1] \sim\{1,1 / 2, \cdots, 1 / k\}$.

(b) $\left\|v_{k}\right\|_{1}<1$ for all $k$,

(c) $\lim _{x \rightarrow 1 / j} v_{k}(x)=\infty$ for $j=1,2, \cdots, k$,

(d) $v_{k}(x)=0$ if $0 \leqq x \leqq 1 /(k+1)$,

(e) $v_{k+1} \geqq v_{k}$.

Assume $0 \leqq w \leqq g$ can be found with $\rho(g-w)<1 / 2$ and $\left\{\beta_{k}: k=1,2, \cdots\right\}$ can be found so that if $0 \leqq f \leqq w$ and $f \leqq \alpha v_{k}$ for some $k \in N$ then $f \leqq \alpha \beta_{k} g$. Now $\gamma(w)>1 / 2$. Pick $\delta>0$ so that if $x \in(0, \delta]$ then $w(x)=\delta(w) g(x)$ and pick $k$ so that $1 / k<\delta$. Now for every $m \in N$ there is an open interval $0_{m}$ about $1 / k$ so that if $x \in 0_{m}$ then $v_{k}(x) \geqq m g(x)$. Now construct $h$ to be continuous with support in $0_{m}$ such that $h(1 / k)=w(1 / k)$ and $0 \leqq h \leqq w$. Then $h \leqq 1 / m v_{k}$ implies $h \leqq\left(\beta_{k}\right) / m g$ and so $\gamma(w) \leqq \beta_{k} / m$ for all $m \in N$. It follows that $\gamma(w)=0$ which is a contradiction. Thus $(L, \rho)$ does not have the local $\sigma$-property.

EXAMPle 3. $((3) \nRightarrow(5))$. In this example $u_{n} \downarrow_{\rho} 0$ implies $u_{n} \rightarrow 0(r . u$. but $\tau_{\rho} \neq \tau_{r u}$ on every order interval. Let $L$ be the Riesz space of all real-valued continuous functions $f$ on $[1, \infty)$ such that there exists $\alpha=\alpha(f)$ with $f(x)=f(1) / x$ for $x \geqq \alpha$. Let $\rho$ be the maximum norm. Note that the function $u(x)=1 / x$ is a strong unit in this space.

(i) We show that $(L, \rho)$ has property (3), i.e., if $u_{n} \downarrow_{\rho} 0$ then $u_{n} \rightarrow 0(r . u$.$) . So assume u_{n} \downarrow_{\rho} 0$ and let $\varepsilon>0$. Pick $n_{1}$ so that $\rho\left(u_{n_{1}}\right)<\varepsilon$. Then $u_{n_{1}}(1)<\varepsilon$ and by definition there exists $\alpha \geqq 1$ such that if $x \geqq \alpha$ then $u_{n_{1}}(x)<\varepsilon / x$. Hence if $n \geqq n_{1}$ then $u_{n}(x)<\varepsilon / x$ for $x \geqq \alpha$. Now pick $n_{2} \geqq n_{1}$ such that if $n \geqq n_{2}$ then $\rho\left(u_{n}\right)<\varepsilon / \alpha$. Then $u_{n_{2}}(x) \leqq \varepsilon / \alpha \leqq \varepsilon / x$ for $x \in[1, \alpha]$. Thus for $n \geqq n_{2}$ we have $u_{n} \leqq \varepsilon / x$ and so $u_{n} \rightarrow 0$ (1/x- unif.).

(ii) We show that $(L, \rho)$ does not have property (5). (Recall $(4) \Leftrightarrow(5)$.) Define $u_{n} \in L$ such that $u_{n}(n)=1 / n, u_{n}(x)=0 \quad$ if $x \notin[n-1, n+1]$, and $0 \leqq u_{n} \leqq 1 / x$ for all $x$ and $n=1,2, \cdots$. Then $u_{n} \rightarrow 0(\rho)$ but no subsequence converges to 0 relatively uniformly. Thus $\tau_{\rho} \neq \tau_{r u}$ on order intervals in $L$.

EXAMPLE 4. $((5) \nrightarrow(6))$. In this example $\tau_{r u}=\tau_{\rho}$ on order intervals 
but not on principal ideals. For each $n \in N$ let $v_{n}$ be the sequence defined by $v_{n}(k)=k^{n}$ for $n=1,2, \cdots$. In the Riesz space of all sequences let $L$ be the ideal generated by $\left\{v_{n}: n \in N\right\}$. We define a Riesz norm $\rho$ on $L$ by setting

$$
\rho(x)=\sum_{k=1}^{\infty} \frac{|x(k)|}{k^{k}} .
$$

(i) In order to show that the norm and relative uniform topologies agree on order intervals it is sufficient to show that if $\left\{u_{n}\right\} \subseteq\left[0, v_{m}\right]$ for some $m$ and $\rho\left(u_{n}\right) \rightarrow 0$ then $u_{n} \rightarrow 0(r . u$.$) . But$ $\rho\left(u_{n}\right) \rightarrow 0$ implies $\lim _{n} u_{n}(k)=0$ for each $k \in N$. Since $v_{m}(k)=$ $1 / k v_{m+1}(k)$, it follows easily that $u_{n} \rightarrow 0\left(v_{m+1}-\right.$ unif.).

(ii) We give a principal ideal $A_{0}$ in $L$ for which the norm and relative uniform topologies do not agree. Let $u$ be the constant sequence $u(k)=1$ for all $k$ and let $A_{0}$ be the principal ideal generated by $u$. Note that we have shown in (i) that $(L, \rho)$ has property $(A, i)$, i.e., $u_{n} \downarrow 0$ implies $\rho\left(u_{n}\right) \rightarrow 0$. Now for each $m \inf \left(n u, v_{m}\right) \uparrow_{n} v_{m}$, so $\left\{\inf \left(n u, v_{m}\right): n=1,2, \cdots\right\}$ is a $\rho$-Cauchy sequence for each $m$.

Let $k$ and $m$ be given. Pick $p_{m}$ so that if $n \geqq p_{m}$ then $\rho\left(\inf \left(n u, v_{m}\right)-\inf \left(p_{m} u, v_{n}\right)\right) \leqq 1 /\left(2^{m+k}\right)$. Now since $v_{m} \notin A_{0}$ there exists $r \in N$ such that $v_{m}(r)>2 p_{m}$. Pick $q_{m}>p_{m}$ such that $\left[\inf \left(q_{m} u, v_{m}\right)-\right.$ $\left.\inf \left(p_{m} u, v_{m}\right)\right](r)>\left(v_{m}(r)\right) / 2$. Let $z_{m}^{k}=\inf \left(q_{m} u, v_{m}\right)-\inf \left(p_{m} u, v_{m}\right)$ for all $k$ and $m$. Note $z_{m}^{k} \in A_{0}, z_{m}^{k} \leqq(1 / 2) v_{m}$ and $\rho\left(z_{m}^{k}\right) \leqq 1 /\left(2^{m+k}\right)$ for all $k$ and $m$. Let $w_{k}=z_{1}^{k}+z_{2}^{k}+\cdots+z_{k}^{k}$, so $\rho\left(w_{k}\right) \leqq 1 /\left(2^{k}\right)$. Hence $w_{k} \rightarrow 0(\rho)$ and $w_{k} \in A_{0}$ for each $k$. But if for some subsequence $w_{k_{j}} \rightarrow 0(r . u$. then $w_{k_{j}} \rightarrow 0 \quad\left(v_{m}\right.$-unif.) for some $m$. However if $k_{j}>m$ then $w_{k_{j}} \npreceq(1 / 2) v_{m}$ and we have a contradiction. Thus $\tau_{\rho} \neq \tau_{r u}$ on $A_{0}$.

EXAMPLE 5. $((6) \nRightarrow(7))$. In this example $L$ has a weak unit, $\tau_{\rho}=\tau_{r u}$ on every principal ideal, but $\tau_{\rho} \neq \tau_{r u}$ on $L$.

Let $L$ be the ideal in $\Pi_{n} \ell_{\infty}$ of all $x$ such that

(a) $\rho(x)=\sup _{n}\left\|x_{n}\right\|<\infty$ (where $\|\cdots\|$ is the supremum norm in $\ell_{\infty}$ ) and

(b) except for finitely many $n$ (depending on $x$ ) $x_{n} \in c_{0}$. Note that if $u \in L$ is defined by $u_{n}(k)=1 / k$ for all $k, n \in N$ then $u$ is a weak unit in $L$.

(i) We show that the norm topology equals the relative uniform topology on principal ideals in $L$. Suppose $\left\{x^{(r)}\right\}$ is a sequence in a principal ideal. Then there exists $m$ such that if $n \geqq m$ we have $x_{n}^{(r)} \in c_{0}$ for all $r$. Passing to a subsequence if necessary we may assume $\sum_{r=1}^{\infty} \rho\left(r x^{(r)}\right)<\infty$. Define $v \in \prod_{n} \ell_{\infty}$ by $v_{n}(r)=\sum_{r=1}^{\infty} r\left|x_{n}^{(r)}\right|$. Since $c_{0}$ is uniformly closed in $\iota_{\infty}$, we have that $w_{n} \in c_{0}$ for all $n \geqq m$ and so $u \in L$. Clearly $\left|x^{(r)}\right| \leqq(1 / r) w$, so $x^{(r)} \rightarrow 0(r . u$. $)$. Thus $\tau_{\rho}=\tau_{r u}$ on 
every principal ideal.

(ii) We give a sequence which is norm convergent to 0 but such that no subsequence converges relatively uniformly to 0 . Thus $\tau_{r u} \neq \tau_{\rho}$ on $L$.

Define $x^{(r)} \in L$ for $r=1,2, \cdots$ by

$$
x_{n}^{(r)}(k)= \begin{cases}1 / r & \text { if } n \leqq r \\ 0 & \text { if } n>r .\end{cases}
$$

Then $\rho\left(x^{(r)}\right)=1 / r \rightarrow 0$, but clearly no subsequence converges relatively uniformly.

EXAMPLE 6. $((7) \Rightarrow(8))$. Let $L$ be the Riesz space of sequences with finite support and let $\rho(x)=\sum_{k=1}^{\infty}|x(k)|$. It is obvious that $\tau_{\rho}=\tau_{r u}$ on principal bands and that $\tau_{\rho} \neq \tau_{r u}$.

Example 7. $((8) \nsim(9))$. Let $(L, \rho)$ be any Riesz space with a strong unit such that $L$ is not complete with respect to the corresponding uniform norm.

This completes the proof of Theorem 3. Before giving the proof of Theorem 4, we give a lemma of Luxemburg.

LEMma 1. If $(L, \rho)$ is a normed Riesz space such that $\bar{A}^{\rho}=A^{\text {ru }}$ for all ideals $A$ in $L$ and $u_{n} \downarrow_{\rho} 0$ then there exists $v_{k} \uparrow$ in $L$ such that

(i) $u_{1} \leqq v_{1}$,

(ii) for every $m$ and $k$ in $N$ there exists $n=n(m, k)$ such that $u_{n} \leqq(1 / m) u_{1}+(1 / k) v_{m}$.

Proof. Let $m \in N$ be fixed and let $A$ be the ideal generated by $\left\{\left((1 / m) u_{1}-u_{n}\right)^{+}: n=1,2, \cdots\right\}$. Then $u_{1} \in \bar{A}^{\rho}$, hence there exists a sequence $\left\{w_{k}\right\}$ in $A$ such that $0 \leqq w_{k} \leqq u_{1}$ for all $r$ and $w_{k} \uparrow u(r . u$.$) .$ Indeed we may assume that for some $v_{m} \in L^{+}$we have $u_{1}-w_{k} \leqq(1 / k) v_{m}$ for $k=1,2, \cdots$. Now also let $r$ be fixed. Then since $\left((1 / m) u_{1}-u_{n}\right)^{+}$ is increasing in $n$, we have that $w_{k}$ belongs to the principal ideal generated by $\left((1 / m) u_{1}-u_{n}\right)^{+}$for some $n=n(m, k)$. Now $w_{r}$ is disjoint from $\left(u_{n}-(1 / m) u_{1}\right)^{+}$for this $n$. Thus for $n=n(m, k)$ since $\left(u_{n}-(1 / m) u_{1}\right)^{+} \leqq u_{n} \leqq u_{1}=w_{k}+\left(u_{1}-w_{k}\right)$ we have $u_{n}-(1 / m) u_{1} \leqq$ $\left(u_{n}-(1 / m) u_{1}\right)^{+} \leqq u_{1}-w_{k} \leqq(1 / k) v_{m}$ and $u_{n} \leqq(1 / m) u_{1}+(1 / k) v_{m}$. Finally we note that we may assume $v_{k} \uparrow$.

Theorem 2 now follows easily from this lemma. Assume $(L, \rho)$ has the $\sigma$-property and for every ideal $A$ we have $\bar{A}^{\rho}=A^{\prime r u}$. If $u_{n} \downarrow_{\rho} 0$ in $L$, we want to show $u_{n} \rightarrow 0(r . u$.$) . So let u_{n} \downarrow_{\rho} 0$ and let 
$v_{k} \uparrow$ be selected as in Lemma 1. By the $\sigma$-property there exist $w \in L^{+}$ and positive scalars $\alpha_{0}, \alpha_{1}, \cdots$ such that $u \leqq \alpha_{0} w$ and $v_{m} \leqq \alpha_{m} w$ for $m=1,2, \cdots$. Now given $\varepsilon>0$ pick $m$ so that $\left(\alpha_{0} / m\right)<\varepsilon / 2$ and then pick $k$ so that $\alpha_{m} / k<\varepsilon / 2$. If $n \geqq n(m, k)$ we have $0 \leqq u_{n} \leqq$ $(1 / m) u_{1}+(1 / k) v_{m} \leqq(\varepsilon / 2) w+(\varepsilon / 2) w=\varepsilon w$. Thus $u_{n} \rightarrow 0$ (w-unif.).

Proof of Theorem 4. Assume $(L, \rho)$ is a normed Riesz space with the local $\sigma$-property and such that for every ideal $A$ in $L$ we have $\bar{A}^{\rho}=A^{\prime r u}$. Let $u_{n} \downarrow_{\rho} 0$. We want to show $u_{n} \rightarrow 0(r \cdot u$.$) .$

Let $v_{k} \uparrow$ be selected as in Lemma 1 . Now by the local $\sigma$-property there exist $w_{1} \in L$ and scalars $\left\{\beta_{k}^{1}: k=1,2, \cdots\right\}$ such that $0 \leqq w_{1} \leqq u_{1}$, $\rho\left(u_{1}-w_{1}\right)<1 / 2$ and if $0 \leqq z \leqq \alpha v_{k}$ then $z \leqq \alpha \beta_{k}^{1} u_{1}$. Again there exist $w_{2} \in L$ and scalars $\left\{\beta_{k}^{2}: k=1,2, \cdots\right\}$ so that $0 \leqq w_{2} \leqq u_{1}-w_{1}$, $\rho\left(u_{1}-w_{1}-w_{2}\right)<1 / 4$ and if $0 \leqq z \leqq w_{2}$ and $0 \leqq z \leqq \alpha v_{k}$ then $z \leqq \alpha \beta_{k}^{2} w_{1} \leqq \alpha \beta_{k}^{2} u_{1}$. Continue this selection process by induction to obtain $\left\{w_{i}: i=1,2, \cdots\right\} \subseteq L^{+}$and $\left\{\beta_{k}^{i}: i, k=1,2, \cdots\right\} \subseteq R^{+}$such that $u_{1}=\sum_{i=1}^{\infty} w_{i}\left(\rho\right.$-convergence) and if $0 \leqq z \leqq w_{i}$ and $z \leqq \alpha v_{k}$ then $z \leqq \alpha \beta_{k}^{i} u_{1}$ for $i=1,2, \cdots$.

Now let $A$ be the ideal generated by $\left\{w_{i}: i=1,2, \cdots\right\}$. Since $u_{1} \in \bar{A}^{\rho}$ there exists a sequence $\left\{y_{r}\right\}$ in $A$ so that $y_{r} \rightarrow u_{1}(q$-unif.) for some $q \in L^{+}$. We may assume $u_{1}-y_{r} \leqq(1 / r) q$ for $r=1,2, \cdots$. For each $r$ we have $y_{r} \in A$ so there exist a positive scalar $M_{r}$ and $p_{r} \in N$ such that $y_{r} \leqq M_{r}\left(w_{1}+w_{2}+\cdots+w_{p_{r}}\right)$. Let $\gamma_{r}=\max \left(\beta_{r}^{i}: i=1,2, \cdots, p_{r}\right)$ and pick $k_{r} \in N$ so that $\left(p_{r} \gamma_{r}\right) / k_{r}<1 / r$. By Lemma 1 there exists $n$ satisfying $u_{n} \leqq(1 / r) u_{1}+\left(1 / k_{r}\right) v_{r}$. By the Riesz Decomposition Property we may write $u_{n}=a_{n}+b_{n}$ where $0 \leqq a_{n} \leqq(1 / r) u_{1}$ and $0 \leqq b_{n} \leqq\left(1 / k_{r}\right) v_{r}$. Again $b_{n} \leqq u_{n} \leqq u_{1}$ so $b_{n} \leqq\left(u_{1}-y_{r}\right)+M_{r} w_{1}+\cdots+M_{r} w_{p_{r}}$. Hence $b_{n}=b_{n}^{0}+b_{n}^{1}+\cdots+b_{n}^{p_{r}}$ where $b_{n}^{0} \leqq u_{1}-y_{r} \leqq(1 / r) q$ and $b_{n}^{i} \leqq M_{r} w_{i}$ for $i=1,2, \cdots, p_{r}$. Now $\left(1 / M_{r}\right) b_{n}^{i} \leqq w_{i}$ and $\left(1 / M_{r}\right) b_{n}^{i} \leqq\left(1 /\left(M_{r} k_{r}\right)\right) v_{r}$ imply that $\left(1 / M_{r}\right) b_{n}^{i} \leqq\left(\beta_{r}^{i}\right) /\left(M_{r} k_{r}\right) u_{1} \leqq\left(\gamma_{r}\right) /\left(M_{r} k_{r}\right) u_{1}$ for $i=1,2, \cdots, p_{r}$. Thus $b_{n}^{i} \leqq\left(\gamma_{r} / k_{r}\right) u_{1}$ for $i=1,2, \cdots, p_{r}$. Hence $u_{n} \leqq(1 / r) u_{1}+(1 / r) q+$ $\left(p_{r} \gamma_{r}\right) / k_{r} u_{1} \leqq(1 / r)\left(2 u_{1}+q\right)$. It follows that $u_{n} \rightarrow 0(r . u$.$) .$

Note that the local $\sigma$-property is not enough to conclude $(3) \Rightarrow(5)$. Example 3 shows this since the Riesz space in that example has a strong unit.

Proof of Theorem 5. (i) We first show that the $\sigma$-property implies the local $\sigma$-property. Assume $(L, \rho)$ is a normed Riesz space such that $L$ has the $\sigma$-property and let $u \in L^{+}, v_{k} \uparrow$ and $\varepsilon>0$ be given. We have to show there exist $w \in L$ and a scalar $\beta_{k}>0$ for each $k$ such that $0 \leqq w \leqq u, \rho(u-w)<\varepsilon$, and if $0 \leqq z \leqq w$ and if $z \leqq \alpha v_{k}$ for some $k \in N$ then $z \leqq \alpha \beta_{k} u$. By the $\sigma$-property we have that there exist $v \in L^{+}$and scalars $\delta_{k}>0$ for each $k$ so that $\delta_{k} v_{k} \leqq v$. Now 
pick a small positive scalar $\lambda$ so that if $w=(u-\lambda v)^{+}$then $\rho(u-w)<\varepsilon$. Then if $0 \leqq z \leqq w$ and $z \leqq \alpha v$ it follows that $z \leqq(\alpha / \lambda) u$. So if $0 \leqq z \leqq w$ and $z \leqq \alpha v_{k}$, we have $z \leqq \alpha / \delta_{k} v$ and hence $z \leqq \alpha /\left(\delta_{k} \lambda\right) u$. Thus we can let $\beta_{k}=1 /\left(\delta_{k} \lambda\right)$ for $k=1,2, \cdots$.

(ii) Assume now that in the $\rho$-completion of $L$ every lower element is an upper element. Again let $u, v_{k} \uparrow$ and $\varepsilon>0$ be given. As above we have to show there exist $w \in L$ and scalars $\left\{\beta_{k}: k \in N\right\}$ with the appropriate properties.

We first note that $\lim _{\lambda \rightarrow 0^{+}} \rho\left(u-\left(u-\lambda v_{1}\right)^{+}\right)=0$. Pick a positive integer $m_{1}$ such that $\rho\left(u-\left(u-1 / m_{1} v_{1}\right)^{+}\right)<\varepsilon / 4$. Now set $u_{0}=u$ and $u_{1}=\left(u-1 / m_{1} v_{1}\right)^{+}$. Then if $0 \leqq z \leqq u_{1}$ and $z \leqq \alpha v_{1}$, we have $z \leqq m_{1} \alpha u$. Now proceeding by induction, we obtain a positive decreasing sequence $\left\{u_{n}\right\}$ in $L$ and a sequence of positive integers $\left\{m_{n}\right\}$ so that $\rho\left(u_{n}-u_{n-1}\right)<$ $\varepsilon /\left(2^{n+1}\right)$ and if $0 \leqq z \leqq \alpha v_{n}$ and $z \leqq u_{n}$ then $z \leqq m_{n} \alpha u_{n-1} \leqq m_{n} \alpha u$. Now $\left\{u_{n}\right\}$ is a decreasing $\rho$-Cauchy sequence, so by assumption there exists an increasing $\rho$-Cauchy sequence $\left\{y_{n}\right\}$ such that $\rho\left(u_{n}-y_{n}\right) \rightarrow 0$. Since $\rho\left(u-u_{n}\right)<\varepsilon / 2$ for all $n$, there exists $n_{0}$ such that $\rho\left(u-y_{n_{0}}\right)<\varepsilon$ and $y_{n_{0}} \leqq u_{n}$ for all $n$. Then we may set $w=y_{n_{0}}$ and $\beta^{k}=m_{k}$ for $k=1,2, \cdots$.

We include here examples to show that for a normed Riesz space $(L, \rho)$, the $\sigma$-property and the property that every lower element in the $\rho$-completion is also an upper element are independent.

EXAMPLE 8. Let $L$ be the Riesz space of continuous functions on $[0,1]$ and let $\rho$ be the $L_{1}$-norm. Then $L$ has the $\sigma$-property since it has a strong unit but $(L, \rho)$ does not have the property that every lower element in the $\rho$-completion is also an upper element.

Example 9. This is the same as Example 4. Here $(L, \rho)$ is locally complete but it does not have the $\sigma$-property, since the particular sequence $\left\{v_{n}\right\}$ is not contained in a principal ideal.

REMARKs. 1. Note that if $L$ has a strong unit $u$ then if $\tau_{\rho}=\tau_{r u}$ on order intervals we have $\tau_{\rho}=\tau_{r u}$ on $L$, i.e., $(5) \Rightarrow(8)$. To see this let $\rho_{u}$ be the uniform norm on $L$ generated by $u$, i.e., $\rho_{u}(x)=$ $\inf \{\lambda:|x| \leqq \lambda u\}$. Now suppose $\left\{f_{n}\right\}$ is a sequence such that $\rho\left(f_{n}\right) \rightarrow 0$ but $\rho_{u}\left(f_{n}\right) \nrightarrow 0$. Indeed we may assume $\rho_{u}\left(f_{n}\right) \geqq n$ for each $n$. Then $\left[1 / \rho_{u}\left(f_{u}\right)\right] f_{n} \rightarrow 0(\rho)$ and the sequence $\left[1 /_{\rho_{u}}\left(f_{n}\right)\right] f_{n}$ is order-bounded, so there exists a subsequence which converges uniformly to 0 , a contradiction. Thus every $\rho$-convergent sequence is order-bounded and the result follows.

2. If $L$ has the $\sigma$-property then $\tau_{\rho}=\tau_{r u}$ on principal ideals 
L. C. MOORE, JR.

implies $\tau_{\rho}=\tau_{r u}$ on $L$, i.e., $(7) \Rightarrow(9)$, since every sequence is contained in a principal ideal.

\section{REFERENCES}

1. W. A. J. Luxemburg, Notes on Banach function spaces, XVI, Proc. Acad. Sci. Amsterdam, A68 (1965), 646-667.

2. W. A. J. Luxemburg and A. C. Zaanen, Riesz Spaces I, Amsterdam, North Holland 1971.

Received October 8, 1976.

DUKE UNIVERSITY

DURHAM. NC 27706 


\section{PACIFIC JOURNAL OF MATHEMATICS}

\section{EDITORS}

RICHARD ARENS (Managing Editor)

University of California

Los Angeles, California 90024

C. W. CURTIS

University of Oregon

Eugene, OR 97403

C. C. MOORE

University of California

Berkeley, CA 94720

\section{J. DugundJI}

Department of Mathematics University of Southern Californıa Los Angeles, California 90007

R. FinN AND J. Milgram Stanford University Stanford, California 94305

\section{ASSOCIATE EDITORS}

E. F. BECKENBACH

B. H. NEUMANN

F. WOLF

K. YOSHIDA

\section{SUPPORTING INSTITUTIONS}

UNIVERSITY OF BRITISH COLUMBIA CALIFORNIA INSTITUTE OF TECHNOLOGY UNIVERSITY OF CALIFORNIA MONTANA STATE UNIVERSITY UNIVERSITY OF NEVADA, RENO NEW MEXICO STATE UNIVERSITY OREGON STATE UNIVERSITY UNIVERSITY OF OREGON OSAKA UNIVERSITY
UNIVERSITY OF SOUTHERN CALIFORNIA STANFORD UNIVERSITY UNIVERSITY OF TOKYO UNIVERSITY OF UTAH WASHINGTON STATE UNIVERSITY UNIVERSITY OF WASHINGTON AMERICAN MATHEMATICAL SOCIETY NAVAL WEAPONS CENTER 


\section{Pacific Journal of Mathematics}

\section{Vol. 71, No. $1 \quad$ November, 1977}

Charalambos D. Aliprantis and Owen Sidney Burkinshaw, On universally complete Riesz spaces ............................. 1

Stephen Richard Bernfeld and Jagdish Chandra, Minimal and maximal solutions of nonlinear boundary value problems .................

John H. E. Cohn, The length of the period of the simple continued fraction of

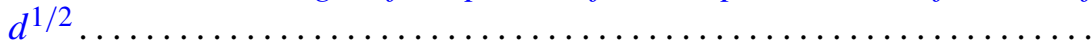

Earl Vern Dudley, Sidon sets associated with a closed subset of a compact abelian group .................................... 33

Larry Finkelstein, Finite groups with a standard component of type $J_{4} \ldots \ldots$

Louise Hay, Alfred Berry Manaster and Joseph Goeffrey Rosenstein, Concerning partial recursive similarity transformations of linearly ordered sets .......................................

Richard Michael Kane, On loop spaces without $p$ torsion. II ............

William A. Kirk and Rainald Schoneberg, Some results on

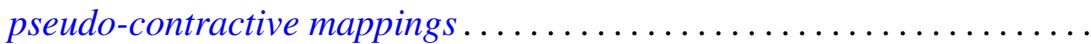

Philip A. Leonard and Kenneth S. Williams, The quadratic and quartic character of certain quadratic units. I. .

Lawrence Carlton Moore, A comparison of the relative uniform topology and the norm topology in a normed Riesz space .................

Mario Petrich, Maximal submonoids of the translational hull 119

Mark Bernard Ramras, Constructing new R-sequences . . .

Dave Riffelmacher, Multiplication alteration and related rigidity properties

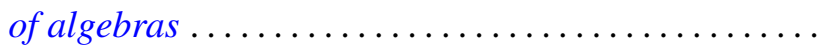

Jan Rosiński and Wojbor Woyczynski, Weakly orthogonally additive functionals, white noise integrals and linear Gaussian stochastic processes.

Ryōtarō Satō, Invariant measures for ergodic semigroups of operators

Peter John Slater and William Yslas Vélez, Permutations of the positive integers with restrictions on the sequence of differences...

Edith Twining Stevenson, Integral representations of algebraic cohomology classes on hypersurfaces ........................

Laif Swanson, Generators of factors of Bernoulli shifts . 